\title{
A membrane filter method for correlating colposcopic, histological, and cytological pictures: The application to nuclear DNA measurements
}

\author{
G. P. BURN, A. SINGER ${ }^{1}$, AND A. I. SPRIGGS \\ From the Nuffield Department of Clinical Biochemistry, the Nuffield Department of Obstetrics and \\ Gynaecology, University of Oxford, and the Laboratory of Clinical Cytology, Churchill Hospital, Oxford
}

SYNOPSIS A method is described for collecting cells on membrane filters in their natural topographical relationships, so that they can be correlated with exact areas in the cervix and with punch biopsy specimens. The same cell sample, after study with Papanicolaou stain, can then be used for other methods without the cells losing their spatial relationships. An example is given of an early cervical carcinoma in which, using a photographic method with a simple type of photometric device, the nuclear DNA was measured from accurately identified normal and neoplastic areas.

The traditional Ayre cervical smear is well adapted for detecting unsuspected cervical disease. For the study of the abnormal cervix, however, it has the defect of providing a cell mixture from the whole area sampled, which usually includes epithelia of several different types. It is useful to take cell samples which maintain the original topography, and this can be done by the method to be described. The areas studied can be correlated with the corresponding colposcopic and histological findings.

Evans (1967) and Evans, McCormack, Sanerkin, Ponsford, and Jones (1969) introduced a method of sampling the whole surface of the living cervix by placing a circular membrane filter by means of a cylindrical applicator. The membrane was then fixed, stained, and mounted. This resulted in a more complete sampling than the older method of Trifon (1958) and Nyklíček (1960) who applied glass slides directly to the cervix. Because of the variable and complex contours of the cervix, we have preferred to apply a number of separate pieces of membrane filter, noting their orientation by means of drawings and photographs.

This method can be used for many purposes. We give as an example the application to nuclear DNA measurements, using photometry of photographic negatives made from Feulgen-stained nuclei.

'Present address: Department of Obstetrics and Gynaecology, Jessop Hospital, Sheffield

Received for publication 9 April 1974.

\section{Material}

Membrane samples were obtained at the time of colposcopy from 200 women. Of these, 125 were attending six to eight weeks after therapeutic termination of pregnancy, 24 were being admitted to a penal institution (Singer, 1972), and 51 were hospital patients, usually attending on account of abnormal cervical smears.

\section{Technique}

MEMBRANE FILTER SAMPLES

Pieces of Metricel (Gelman) membrane, $5 \mu$ pore size, are cut to have an area of approximately 2 or $2.5 \mathrm{sq} \mathrm{cm}$ and of such a shape that there is only one orientation congruent with a predetermined diagram. We have mainly used a segment of a circle or a rectangle with a cut-off corner, thus:
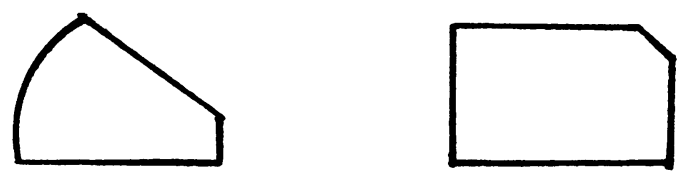

Employing colposcopy, and before the routine application of acetic acid, a piece of membrane is applied to any areas which it is desired to sample, noting or photographing the exact position and 


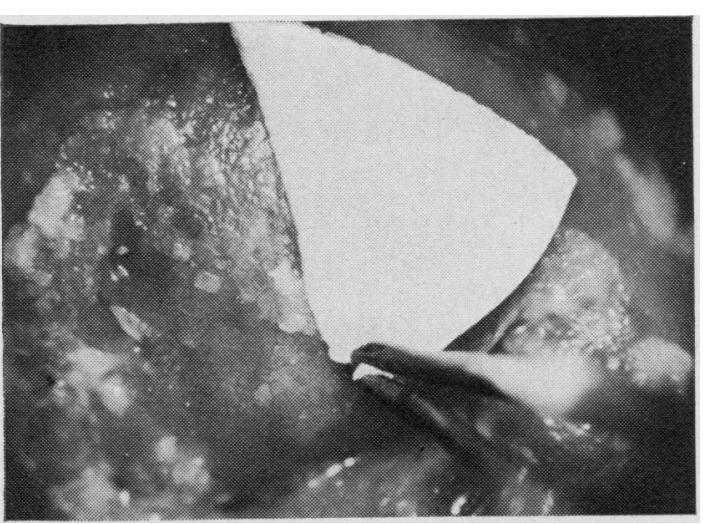

Fig 1 Application of a piece of membrane filter to the living cervix under colposcopic control. $\times 6$

orientation of the membrane (fig 1). It is then peeled off, carrying a layer of cells, and immediately fixed by immersion in industrial methylated spirit.

At any convenient time after fixation, membranes are taken through the standard Papanicolaou stains, ending with xylol, and mounted in Eukitt under coverslips. Histological confirmation of the tissue immediately underlying the membrane was obtained by direct punch biopsy, and, when indicated, by subsequent cone biopsy.

\section{FEULGEN STAINING}

Papanicolaou-stained membranes, after microscopic study and photography, were de-stained by immersion for nine hours in $1 \% \mathrm{HCl}$ in ethanol and restained by the standard Feulgen method, using a hydrolysis time of eight minutes at $60^{\circ} \mathrm{C}$.

\section{METHOD FOR DNA ESTIMATIONS}

These preparations could be measured using any microdensitometric apparatus currently recom- mended for sections or smears. We have applied a modification of the method of Adams (1968), using photographic film and a simple photometric device.

\section{Photography}

Photography of selected fields was performed using Ilford Pan F film, with a Balzer interference filter $573 \mathrm{~nm}$ and a magnification of $\times 160$. The correct exposure and development were determined empirically to give grey levels over all parts of the nucleus, avoiding areas of complete translucency at chromocentres, and to give a background transmittance of between 25 and $50 \%$. All fields were photographed with identical illumination and exposure on a Zeiss photomicroscope with a separate shutter. Light was standardized with an eyepiece photometer.

Theory requires that the exposure shall be adjusted so that the transmittance of the film is inversely proportional to the logarithm of the exposure. This has to be tested on a particular film and using the chosen developer. Logarithmic variation in the exposure is achieved by combining a series of neutral density filters, graded in terms of optical density. Successive photographic exposures were made in which the exposure was so controlled, and the relationship was linear from 100 to $25 \%$ transmittance.

\section{Apparatus}

The apparatus (fig 2) consisted of a film holder, diaphragm, and photometer. The film was illuminated by a lamp whose filament was $70 \mathrm{~mm}$ below the film. An iris diaphragm was situated $6 \mathrm{~mm}$ above the film, it opened to $16 \mathrm{~mm}$ and could be closed to 2.5 $\mathrm{mm}$ against a stop. This was the size needed to encircle the largest nucleus. A silicon photodiode could be brought into line above the iris at a distance of $20 \mathrm{~mm}$ above the film so that the transmitted light could be measured. Alternatively, a $10 \mathrm{~mm}$

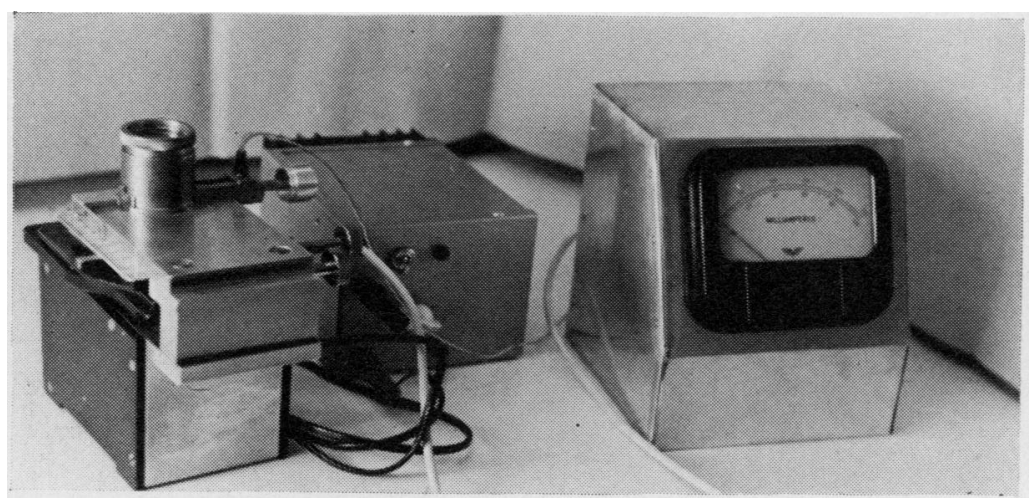

Fig 2 Photometer: left, apparatus for measuring the photographic film; centre, adjustable stabilized power supply; right, photodiode amplifier. 
aperture above the iris enabled the film to be moved about under direct vision until a nucleus could be seen. Observation was improved with a magnifying lens $50 \mathrm{~mm}$ above the film. Movement of the film was obtained by converting a microscope stage having rack and pinion motion in two directions. The lamp was run from an adjustable stabilized supply. The diode current was amplified and recorded on a large meter.

\section{Standardization}

Standardization of the apparatus was obtained by reading over an area of clear film (not background) from a part which had not been exposed to light. The iris was reduced to the limit of $2.5 \mathrm{~mm}$, the photodiode was inserted into the light path and the illumination adjusted till the meter read $100 \%$ transmittance.

\section{Measurements}

To perform measurements, nuclei were brought into view by opening the iris, using the aperture and lens. Nuclei out of focus were rejected. A selected nucleus was positioned in the centre of the iris by means of the mechanical stage. The iris was closed down to the stop. The photodiode was inserted in the light path and the meter reading was noted.

It was necessary to measure the background absorption at two or three places round the nucleus. Several nuclei close together can employ the same group of background readings. Further nuclei 10 $\mathrm{mm}$ away required a separate set of readings. The mean background reading was subtracted from the nucleus reading to give a difference measured in units of transmission which represented the density of the nucleus.

\section{Results and Discussion}

Since the cells are seen in their proper relations to each other, a brief description will be given of the appearances in Papanicolaou-stained membranes.

In Papanicolaou's (1943) original terminology, the cells found in vaginal smears were classified by cell layers-basal, intermediate, and superficial. Since the exfoliated cells can only come from the surface layer (apart from actual ulceration) this system is unsatisfactory. In what follows we will use a terminology similar to that of Reagan (1958) and Patten (1969). The names 'karyopyknotic' and 'intermediate' remain. 'Basal' or 'parabasal' cells are called 'metaplastic' (as suggested in the Acta Cytologica's opinion poll of 1958) since they come from the surface of immature metaplastic epithelium of the transformation zone; they have nothing to do with the basal or parabasal cells of histology, which could not be exfoliated spontaneously into vaginal fluid.

\section{CELLS OF NATIVE EPITHELIUM}

\section{Endocervical columnar cells}

In membrane preparations these cells are often badly shown, partly because of the presence of pus cells in the endocervical mucus, and partly because the very delicate cells suffer from the surface tension forces operating when they are sucked against the porous surface of the membrane. Also, they do not normally exfoliate in layers, and are therefore torn from their setting. They are displayed best when whole pieces of epithelium come off together.

\section{Transitional endocervical cells}

At the squamo-columnar junction one sometimes

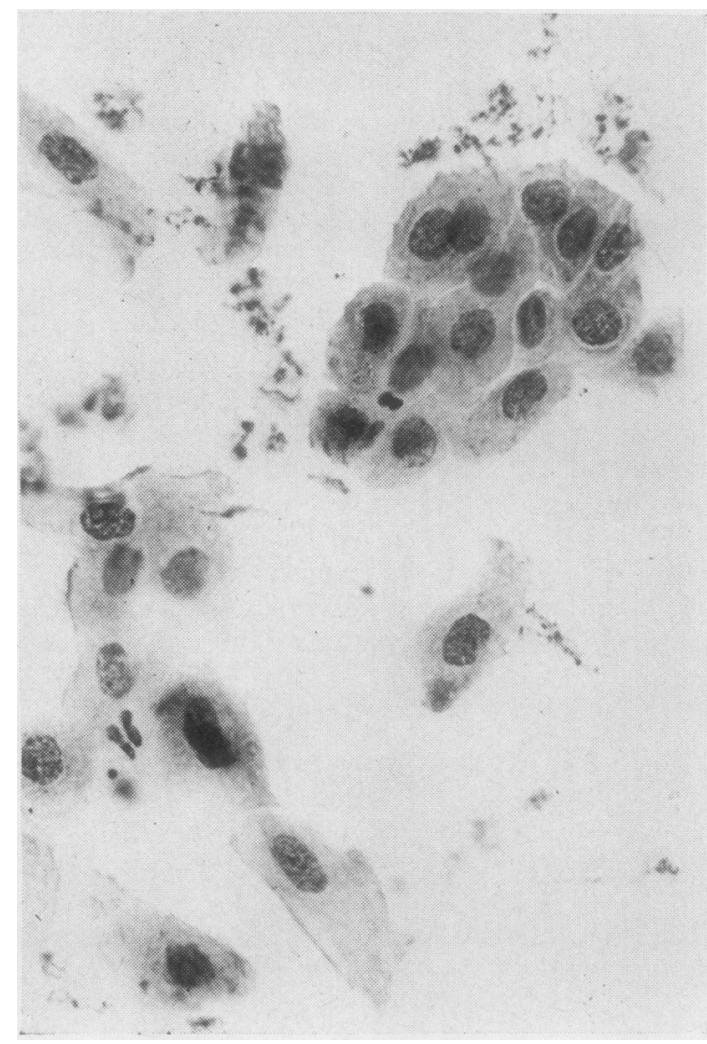

Fig 3 'Transitional' cells at the normal squamocolumnar junction, as seen on a membrane filter preparation. Tinctorially they resemble endocervical columnar cells, but the cytoplasm is more profuse and angular. Papanicolaou $\times 800$. 
sees small areas of endocervical cells showing transitions towards the shape and appearance of intermediate squamous cells (fig 3 ). This appearance is not seen regularly; the commonest junction is represented simply by a gap between columnar and squamous cells, resulting from failure of apposition of the membrane to the depression where the two epithelia join. When found, the cells in transition appear roughly square or triangular, sometimes elongated, but always angular and not rounded. They are pale-staining, with the light basophilia seen in the columnar cells and also in the neighbouring intermediate squamous cells.

\section{Intermediate squamous cells}

The squamous cells nearest to the endocervical epithelium are normally intermediates. In our material this is much the commonest type, and is normal for mature squamous epithelium originating in squamous metaplasia. In 'young' metaplastic epithelium the filter membrane removes a delicate layer of large pale-staining intermediate cells which appear widely spaced. Further removed from the existing squamo-columnar junction there are thicker sheets of intermediate cells.

\section{Karyopyknotic squamous cells}

In our material these were seen infrequently, except in rather small discrete areas scattered over intermediate epithelium.

\section{Atrophic squamous cells}

In some samples from postmenopausal women, sheets of cells are found with rather poorly displayed cytoplasmic borders, and of smaller size than the usual intermediate cells. These sheets, familiar from cervical smears, come off areas of very thin squamous epithelium made up of cells with more or less approximation to cuboidal shape. In smears of atrophic epithelium with little squamous differentiation, there is sometimes confusion with the 'dedifferentiation' of neoplasia. This difficulty gives less trouble in membrane preparations, as the cell relationships are not disturbed and the regularity of cell spacing is preserved.

\section{CELLS OF METAPLASTIC EPITHELIUM}

These show a succession of appearances depending on the maturity of the squamous metaplasia. Often metaplastic cells (formerly known as 'parabasals') cover considerable areas of transformation zone, which is easily identifiable colposcopically (Coppleson and Reid, 1967).

\section{DYSPLASIA AND MORE ADVANCED LESIONS}

\section{Dysplasia}

However mild they may be, dysplastic lesions show 2

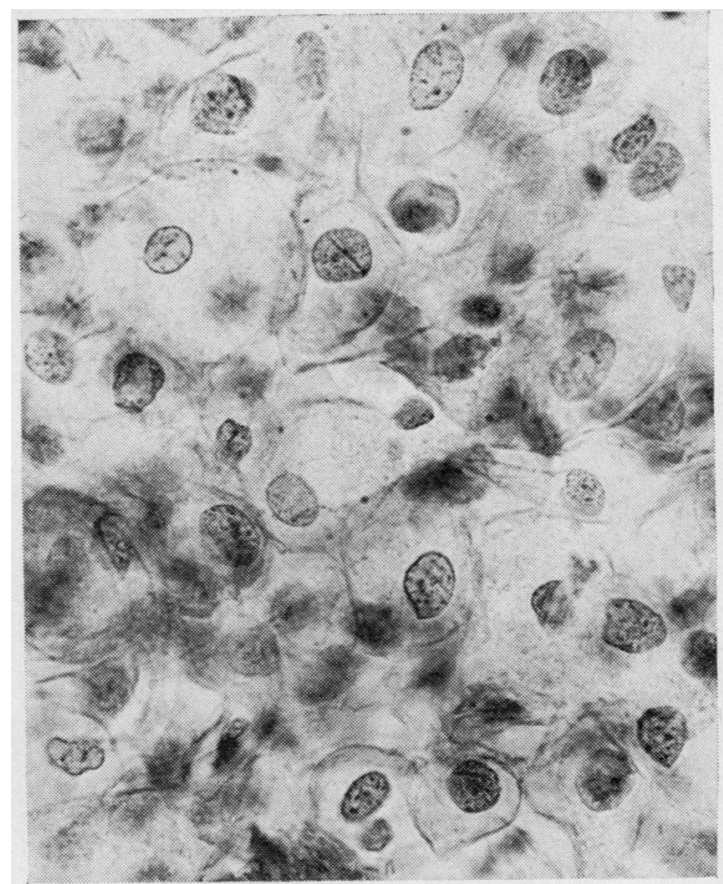

Fig 4 An area of minor dyskaryosis seen in a membrane filter. This corresponded to an area of minimal dysplasia in the histological section. Papanicolaou $\times 800$.

nuclear abnormalities in their surface layers. There are descriptions in the literature suggesting that an epithelium can have neoplastic nuclei in its deeper layers but normal ones at the surface; this is unthinkable because the superficial cells were generated from below. In any case, most cases of dysplasia are detected in the first place by finding large abnormal nuclei in smears.

Figure 4 shows an example of dyskaryotic cells coming from an area of mild dysplasia. The appearances vary from place to place even in the same cervix, and the present technique is valuable in isolating morphologically distinct areas, which may represent clones, for special studies such as nuclear DNA measurements.

\section{Carcinoma in situ and microcarcinoma}

Here the surface layer lacks squamous differentiation, and the millipore shows sheets of relatively uniform cells with nuclear enlargement and lack of polarity.

\section{Invasive carcinoma}

In carcinoma the membrane has little advantage over the conventional smear, since the topography is 


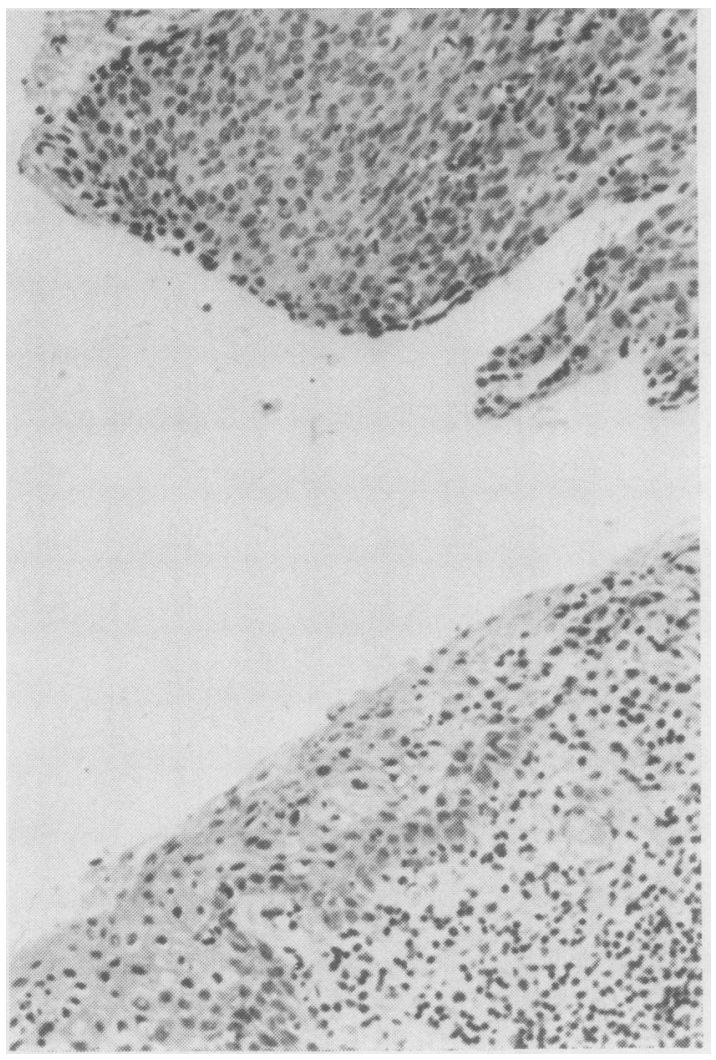

Fig 5

Fig 5 Section of cervix showing (above) an area of carcinoma in situ and (below) adjacent squamous epithelium without neoplastic features. Invasion was present elsewhere in this cervix. $H \& E \times 128$.

Fig 6 Part of the membrane filter topographically corresponding to fig 5, showing neoplastic nuclei above and normal nuclei below. Feulgen stain $\times 250$.

generally clear from inspection. When there is no ulceration, the malignant cells are well shown.

FEULGEN PHOTOMETRY

As an example of the application of the membrane filter technique to nuclear DNA measurement, a section is presented in fig 5 from a cervical biopsy, showing an area of carcinoma in situ (above) and an area of squamous epithelium without malignant features (below). Elsewhere the sections showed early invasive carcinoma. Part of the exactly corresponding membrane filter preparation is shown in fig 6 , stained by the Feulgen procedure. The

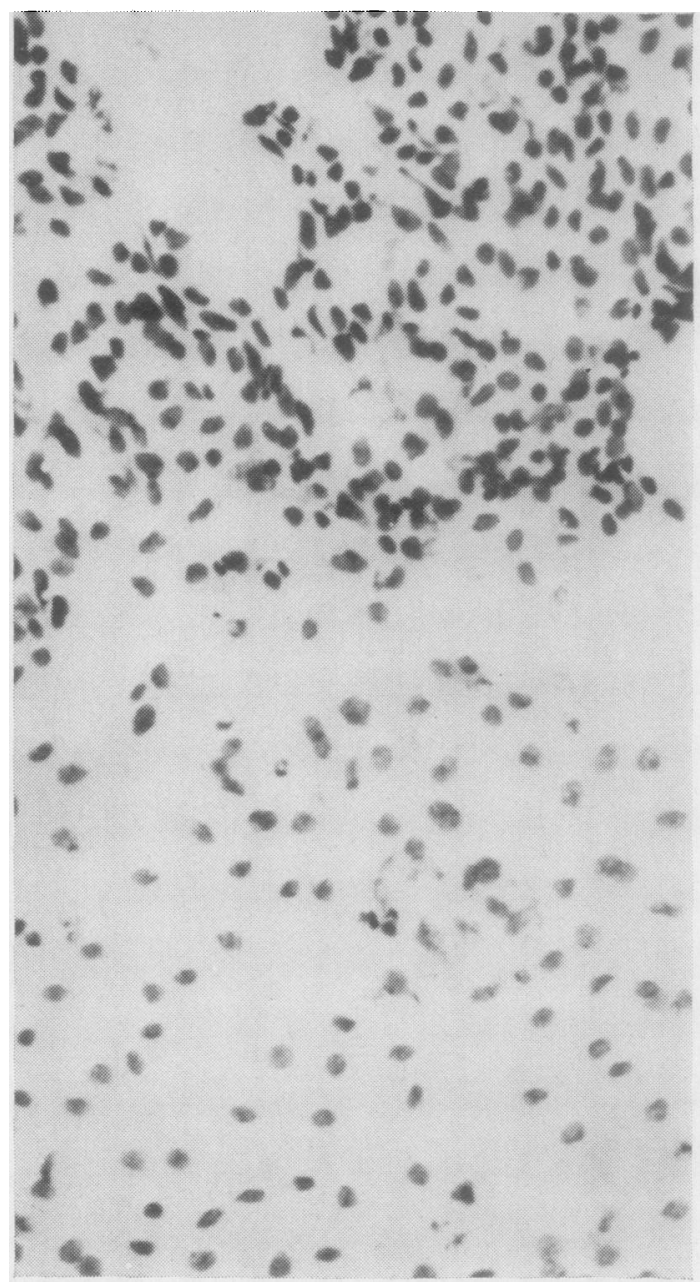

Fig 6 junction is shown between neoplastic cells (above) and normal cells (below).

Separate photographs were made from the two cell populations and these were measured densitometrically. The histograms are shown in figure 7. In 0 spite of the scatter of values (which is also found using more sophisticated apparatus), there is a clear? difference in the position of the modal peak, demonstrating that a new clone has developed. The great advantages of the membrane filter method are seen in these pictures: that superficial cells are sampled which have most probably finished DNA synthesis (at least in the normal areas); that practi- 


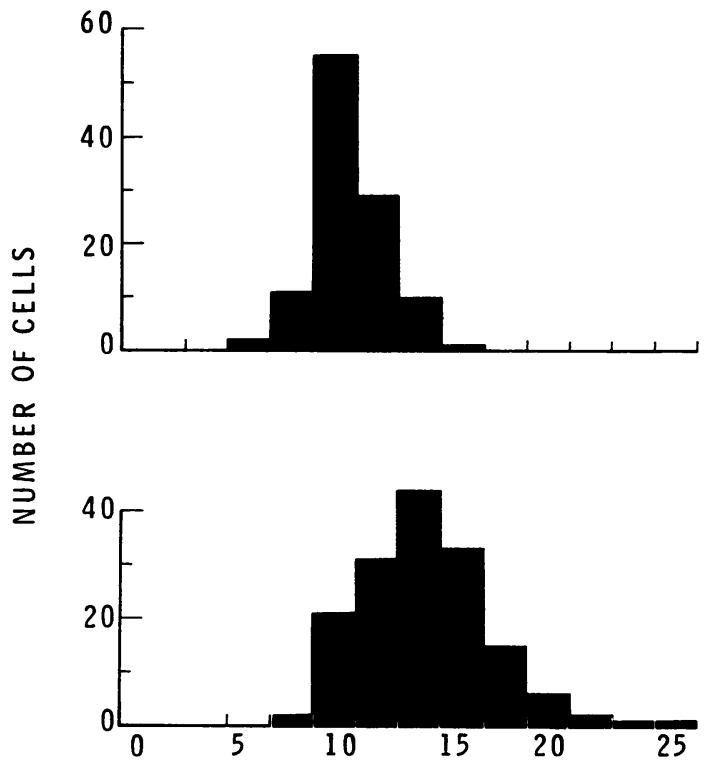

\section{Conclusion}

The membrane method is not presented as a substitute for the cervical smear. Its place is not in the detection of unsuspected disease, but as a research method for studying the epithelium of the cervix. Its main importance is in the application of histochemical and special microscopical techniques to whole areas which may represent distinct clones, and which can in this way be related to the colposcopic appearances and to the corresponding histological sections.

We are grateful to Professor Sir John Stallworthy, in whose department the colposcopies were carried out, for his encouragement and criticism.

\section{References}

Adams, L. R. (1968). A photographic cytophotometric method which avoids distributional error. Acta cytol., 12, 3-8.

Coppleson, M., and Reid, B. (1967). Preclinical Carcinoma of the Cervix Uteri, Oxford, Pergamon Press.

Evans, D. M. D. (1967). Cytological method for assessing the topography of neoplastic cells on the ectocervix. Lancet, 2, 972.

ARB ITRARY UNITS

Fig 7 Histograms of densitometric measurements of normal (upper) and malignant (lower) nuclei. Ordinate: number of nuclei contained in each column. Abscissa: difference between nucleus transmission and background transmission, which is equivalent to DNA in arbitrary units.

cally all the cells in a given area can be assumed to come from a well defined and identified spot; and that both normal and abnormal areas are studied on the same preparation, so that variations in staining operate equally on both cell populations.

Evans, D. M. D., McCormack, J., Sanerkin, N. G., Ponsford, P., and Jones, J. (1969). A membrane cytologic technique for assessing the extent of ectocervical carcinoma using the Tenovus applicator. Acta cytol., 13, 119-121.

Nyklicek, O. (1960). Review of techniques of cervical smears. Acta cytol., 4, 227-228.

Opinion Poll on Cytological Terminology. (1958). Acta cytol., 2, 63139.

Papanicolaou, G. N., and Traut, H. F. (1943). Diagnosis of Uterine Cancer by the Vaginal Smear. New York, Commonwealth Fund.

Patten, S. F. (1969). Diagnostic Cytology of the Uterine Cervix. Basle, Karger.

Reagan, J. W. (1958). The nature of the cells originating in so-called, 'precancerous' lesions of the uterine cervix. Obstet. Gynec. Surv., 13, 157-179.

Singer, A. (1972). A colposcopic study of a high risk group of women. Acta cytol., 16, 392-393.

Trifon, H. M. (1958). Detection and localisation of preclinical carcinoma of the cervix by contact smears. Obstet. Gynec. Surv., 13, 707-717. 